\title{
Morphological and molecular characterization of culturable leaf endophytic fungi from Malabar Spinach: The first report
}

\author{
Moutusi S $\mathbf{1}^{1}$ Buela Parivallal $\mathbf{P}^{2}$, Prasannakumar $\mathbf{M K}^{2}$ and Kiranmayee $\mathbf{P}^{1}$
}

${ }^{1}$ Department of Cell Biology and Molecular Genetics, Sri Devaraj Urs Academy of Higher Education and Research, Kolar - 563 103, Karnataka, India

${ }^{2}$ Department of Plant Pathology, Gandhi Krishi Vignana Kendra, University of Agricultural Sciences, Bangalore 560 065, India

Moutusi S, Buela Parivallal P, Prasannakumar MK, Kiranmayee P 2019 - Morphological and molecular characterization of culturable leaf endophytic fungi from Malabar Spinach: The first report. Studies in Fungi 4(1), 192-204, Doi 10.5943/sif/4/1/21

\begin{abstract}
To isolate and identify the cultivable leaf endophytic fungi of Basella alba L. and B. rubra L., healthy and fresh leaves were chosen and collected from Sri Devaraj Urs Academy of Higher Education and Research college botanical garden. Authenticated materials were made into small bits, surface sterilized by applying the standard protocol and cultured on a potato dextrose agar plate supplemented with appropriate antibiotics, incubated for 7 to10 days at $28^{\circ} \mathrm{C}$. Seed borne fungal endophytes were also identified by keeping scarified seeds on sterilized agarose. A phylogenetic tree was constructed for the fungi of both the plants. A total of 26 and 19 endophytic cultivable fungi were isolated from B. alba and B. rubra respectively. Majority of the endophytes belongs to the genus Alternaria and several genera in class Dothideomycetes. Our preliminary observation indicated that the vertically transmitted endophytic fungi are the dominant endophytes of the selected plants. We conclude that Alternaria is predominant in both the plants. The mechanism of transition of the microbe from being non-pathogenic in its host to being pathogenic in other plants needs to be studied and elucidated.
\end{abstract}

Key words - Basella alba - Basella rubra - CTAB - ITS - phylogenetic tree

\section{Introduction}

Endophytic microbiota includes bacteria and fungi that harbor plants. They are particularly common and highly varied microorganisms that live inside the plants. Their presence is asymptomatic or systemic in the tissues of the plants. Endophytes are basically categorized as vertically and horizontally transmitted ones. The traditional conviction is that these microorganisms and plants are mutualistic and are considered as obligate symbionts. In plants, the endophyte number ranges from one to hundreds or thousands of species. However, endophytes are habitually neutral or sometimes pathogenic, depending on the genotype of the host plant, the endophyte and the environment (Faeth \& Fagan 2002).

Endophytic fungi colonize either in intercellular or intracellular spaces of plant parts like, leaves, stem, flowers, fruits, seeds and roots. These fungi can either complete or part of their life cycle inside the host tissue. The host plants show higher nutrient uptake, get immense resistance to herbivores, abiotic stresses (Mishra et al. 2014). 
The widely accepted molecular marker for endophytic fungal barcoding is the Internal Transcribed Spacer (ITS) ribosomal Deoxyribo Nucleic Acid (rDNA). The ITS1, ITS4 and the highly conserved 5.8S exon gene of rDNA are the three regions of ITS (Fig. 1).

The beneficial microorganisms are categorized as endophytes (Sushanto et al. 2016). Under certain circumstances, these endophytes may change to parasites and vice versa. By taking the advantage, endophytes complete their entire life cycle with the respective hosts. Endophytes gain their nutrition from the host and in turn, with mutualism, host plants enhance ability to withstand adverse environmental conditions, resistance to herbivores and pathogens by gaining kind of bioactive compound/compounds, making the host plant competitive enough in all the aspects of its life cycle (Jia et al. 2016). Seeds are dormant till they get suitable growth conditions. Seed borne microbes are thought to help and promote seed germination in soil (Rodríguez et al. 2018).

These microbes are important as they pass through generations by vertical transmission route. During this process, the pathogenic microbes become weak, and this mutualism provides support the host plant to grow, defend and develop. In turn, host plant helps microbial proliferation and provides valuable endosymbiont offsprings (Frank et al. 2017).

Almost all the plant species are known to have properties of either antibacterial, antiproliferative, antidiabetic, antiallergic etc. Wide range of availability, cost effectiveness and less toxicity than synthetic drugs make plants the most considerate to the scientific community. Incidentally, many plants in nature harbor certain kinds of microorganisms in them, which are both beneficial and harmful to the host plant (Sushanto et al. 2016).

The selected plants, Basella alba L and B. rubra L belong to Basellaceae. These are perennials, found in tropical Asian and African countries and have been used from the ancient times to cure various diseases, namely anticancer, antiviral, antioxidant, anti-inflammatory, anticholesterol, anti-ulcer, antimicrobial, anti-hypoglycemic, wound healing, androgenic (Shade et al. 2017).

In Ayurveda, these plants are being used as laxative, in the treatment of skin diseases, hemorrhages, diarrhea, dysentery etc. These plant species contain betacyanin, carotenoids, bioflavonoids, $\beta$-sitosterol and lupeol which are reported to have antioxidant, antiproliferative, antimicrobial, anti-inflammatory activities etc. Reasonable attention has been given to endophytes than their host plants. Alike all other plants, the selected plant species also harbor endophytes, whose identity and functional properties of the fungal extracts have been the gaps to be filled. Therefore, we tried to identify the endophytes.

The idea of the present investigation is to isolate and identify the cultural leaf endophytic fungi of B.alba and B. rubra.

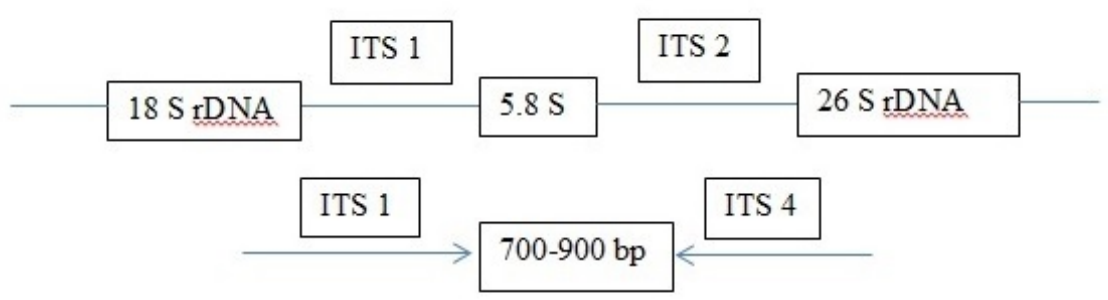

$18 \mathrm{~S}, 5.8 \mathrm{~S}$, and $26 \mathrm{~S}===$ Coding regions

Fig. 1 - Internal Transcribed Spacer (ITS) regions of rDNA

\section{Materials and methods}

\section{Sample collection}

Experiments were conducted in Department of Cell Biology and Molecular Genetics, Sri Devaraj Urs Academy of Higher Education and Research (SDUAHER), Kolar and Department of 
Plant Pathology, University of Agriculture Sciences, GKVK (Gandhi Krishi Vignana Kendra), Bangalore. Healthy and fresh leaves of $B$. alba and B. rubra were chosen and collected from Dhanvanthari botanical garden of the SDUAHER, Kolar. The plant samples were identified, authenticated and voucher specimens were submitted to Department of Forestry and Environmental Science, University of Agriculture, GKVK, Bangalore. The specimen number (UASB-4590) has been given with collection numbers: MS 01 and MS 02. In recent observations, both plants have been declared as same with a difference of appearance in color. By taking this into consideration, we would like to study and compare the prevalence of endophytic fungi in both the plants.

\section{Isolation of endophytes}

The plant samples were surface sterilized by washing under running tap water. Under aseptic conditions, the materials were made into small bits, surface sterilized with detergent water, $2 \%$ sodium hypo chlorite, ethanol $(20 \%, 50 \%, 70 \%, 100 \%)$ followed by autoclaved distilled water for 2-3 times. After wiping on tissue paper for removal of excess water, these bits were placed on a potato dextrose agar (PDA) plate supplemented with $50 \mathrm{mg} / \mathrm{L}$ of Streptomycin and $35 \mathrm{mg} / \mathrm{L}$ of Ampicillin to suppress the bacterial growth. Sterilized needle was used to prick the bits for proper emergence of fungi. The plates were incubated for 7-10 days at $28^{\circ} \mathrm{C}$. The surface sterilization efficiency was checked by plating the last wash onto nutrient medium (http: //dx. doi.org/ 10.17504/protocols.io.jhmcj46).

Colony frequency is equal to the number of segments colonized by a single endophyte divided by the total number of segments observed, multiplied by 100 . The following formula will give the \% colonization.

$$
\text { \% Colonization }=\frac{\text { Number of segments yielded endophytes }}{\text { Total number of segments incubated }} \quad \text { X100 }
$$

Single microbial cultures were obtained through sub-culture. A single fungal isolate from the master plate was sub cultured by hyphal tip method and incubated on respective media for the required time period. Morphologically, the fungal culture purity was determined. The pure form of all the fungi were streaked on a slant, preserved as glycerol stock and deposited in department of Pathology, University of Agricultural Sciences, Gandhi Krishi Vignana Kendra (GKVK). Each organism was labeled with code numbers, part used with date of isolation. Replications were maintained for identifying the organisms by staining, and for further utility of the organisms.

\section{Genotypic and phenotypic identification of fungi}

Initially, observed the colonies under microscope, noted the texture, colour, the dimensions and morphology of hyphae and conidia and the reproductive features. The standard CTAB (Cetyl Trimethyl Ammonium Bromide) method was used to isolate DNA from all the fungi (Heinig et al. 2013). For molecular biological identification, PCR was performed by using ITS 1 (forward) and ITS 4 (reverse). After checking the concentration of DNA in nanodrop, $0.1 \mu \mathrm{g}$ of fungal DNA was used for Polymerase Chain Reaction (PCR) amplification with ITS1 (forward 5'TCCGTAGGTGAACCTGCGG-3') and ITS4 (reverse 5'-TCCTCCGCTTATTGATATGC-3') by adopting $94^{\circ} \mathrm{C}$ for $5 \mathrm{~min}, 35$ cycles of $94^{\circ} \mathrm{C}$ for $1 \mathrm{~min}, 53^{\circ} \mathrm{C}$ for $1 \mathrm{~min}, 72^{\circ} \mathrm{C}$ for $1 \mathrm{~min}, 72^{\circ} \mathrm{C}$ for $7 \mathrm{~min}$, PCR conditions. The PCR mixture included $15 \mu \mathrm{L}$ of Taq polymerase master mix, $1.5 \mu \mathrm{L}$ each primer $(10 \mathrm{pmol} / \mu \mathrm{L}), 3 \mu \mathrm{L}$ template DNA, and $9 \mu \mathrm{L}$ molecular biology grade water. The amplified product was loaded in $1.0 \%$ agarose gel with $1 \mathrm{~kb}$ DNA ladder. The amplified PCR products were submitted to obtain sequences to Eurofins Genomics, Germany on 3730xl/3730XLPC-19137-017, Applied Biosystems (ABI), United States of America by using big dye terminator. Aligned the attained sequences and compared with GenBank databases (National Centre for Biotechnology Information website) by Basic Local Alignment Search Tool (BLAST) tool (http://www.ncbi.nlm.nih.gov). The sequences were known by BLAST and GenBank helped us to 
know the highly similar organisms by taking the sequence homology. The top three similar hits from BLAST with 98\% similar to query sequence were considered and the name was assigned to the isolated fungi. The obtained fungal rDNA sequences with the name of the organism were deposited to GenBank and the ITS accession numbers were listed.

\section{Phylogenetic tree and Neighbor-joining algorithm}

The resulted sequences and the nearest identified sequence based on BLAST search were used to make phylogenetic analysis, also calculated the neighbour-joining algorithm. Phylogenetic tree for both the plants have been built for a wide range of organisms using sequences (gene), in particular, by the identification of orthologous genes that have been vertically inherited. The DNA sequences obtained were used as a wide range in order to classify the orthologous genes that are vertically been inherited.

A phylogenetic tree was fabricated for both B. alba and B. rubra individually using an online tool clustal omega. The tree was constructed as neighbour-joining (NJ) tree (Tamura et al. 2011).

\section{Seed borne fungal endophytes}

Endophytic fungi display a wide-range in mode of transmission, in particular, vertical transmission. In finding out seed borne endophytic population, a methodology has been developed and the healthy seeds of both the plants were selected, scarified, and surface sterilized by adopting the same protocol followed for the isolation of leaf endophytes. Aseptically, one seed per tube was transferred to an autoclaved $0.8 \%$ agarose medium. Quadruplicates were maintained for each plant. The tubes were kept under dark condition for 10 days, shifted to light at room temperature for about a month. Fungal mycelium and spores were stained with lactophenol cotton blue.

\section{Results}

\section{Isolation and identification of endophytic fungi}

Out of 24 and 12 leaf bits, a total of 26 and 19 endophytic cultivable fungi were isolated from B. alba and B. rubra respectively (Fig. 2).

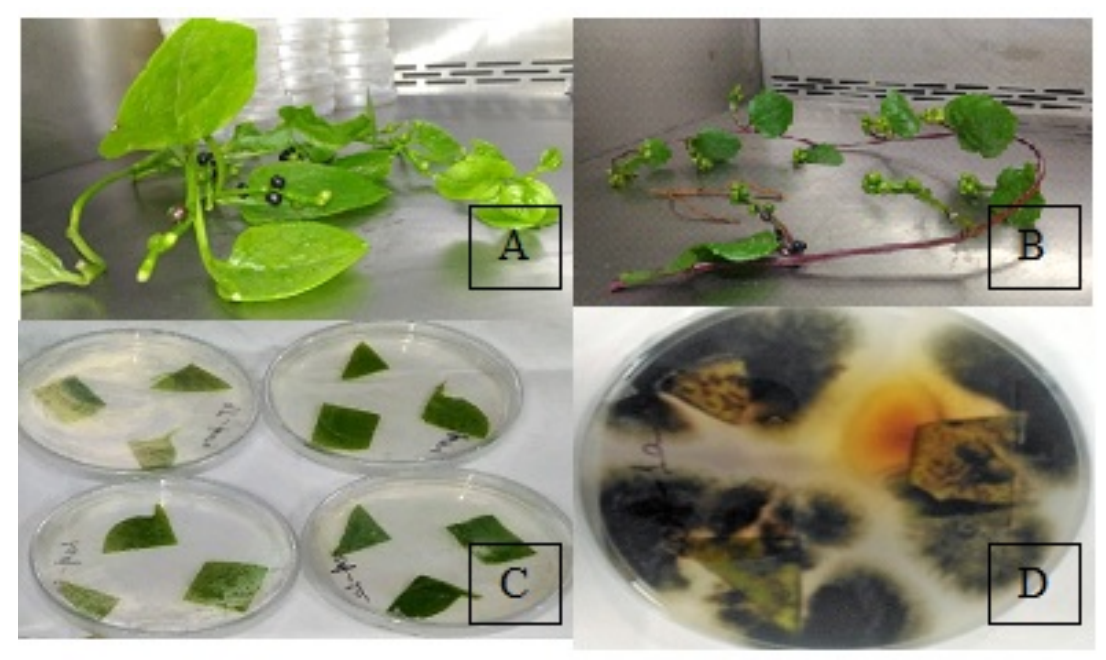

Fig. 2 - Fresh plant material and the plant parts on artificial medium. A, B Basella alba and Basella rubra, respectively from herbal garden in a laminar air flow. C Surface sterilized plant bits. D Colony of endophytic fungi on PDA.

After obtaining sequencing results, the isolates belong predominantly to Alternaria species, followed by Ascomycota spp., Cladosporium cladosporioides, Microdiplodia spp., Pleosporales spp., and Stemphylium lycopersici. Colony frequency was calculated and the percentage of each fungus was given in Table 1. 
Table1 Colony frequency percentage of each fungus isolated from the leaves of $B$. alba and $B$. rubra.

\begin{tabular}{|c|c|c|c|c|}
\hline Organisms & $\begin{array}{l}\text { CF\% of } \\
\text { BA }\end{array}$ & $\begin{array}{l}\text { CF\% of } \\
\text { BR }\end{array}$ & Division & $\begin{array}{l}\text { Nature of the organism in host } \\
\text { (Life form) }\end{array}$ \\
\hline Alternaria alternata & 37.5 & 33.33 & Ascomycota & Major plant pathogen \\
\hline Alternaria arborescens & 8.33 & - & Ascomycota & Plant pathogen \\
\hline Alternaria brassicae & 4.17 & - & Ascomycota & Plant pathogen \\
\hline Alternaria brassicicola & - & 8.33 & Ascomycota & Plant pathogen \\
\hline Alternaria longipes & 4.17 & - & Ascomycota & Endophyte and pathogen \\
\hline Alternaria sp. & 12.5 & 33.33 & Ascomycota & Major plant pathogen \\
\hline Alternaria tenuissima & 16.67 & 25 & Ascomycota & $\begin{array}{l}\text { Saprophytic and opportunistic } \\
\text { plant pathogen }\end{array}$ \\
\hline Ascomycota sp. & - & 8.33 & Ascomycota & Endophyte \\
\hline $\begin{array}{l}\text { Cladosporium } \\
\text { cladosporioides }\end{array}$ & - & 8.33 & Ascomycota & Endophyte and pathogen \\
\hline Microdiplodia sp. & 4.17 & - & Ascomycota & Endophyte \\
\hline Pleosporales sp. & 12.5 & 25 & Ascomycota & Endophyte \\
\hline Stemphylium lycopersici & 4.17 & 8.33 & Ascomycota & Endophyte and pathogen \\
\hline Stemphylium sp. & 4.17 & - & Ascomycota & Endophyte and pathogen \\
\hline
\end{tabular}

CF: Colony Frequency; BA: B. alba; BR: B. rubra

The following were the fungi associated with the selected plants of the present study: total of six Alternaria species, Ascomycota spp., Cladosporium cladosporioides, Microdiplodia spp., Pleosporales spp., Stemphylium lycopersici. Our study illustrates that the majority of the endophytes from $B$. alba and $B$. rubra belongs to Alternaria and several genera in class Dothideomycetes.

\section{Identification of endophytic fungi by DNA sequencing}

Morphologically, all the cultures were stained for identification (Figs 3, 4).

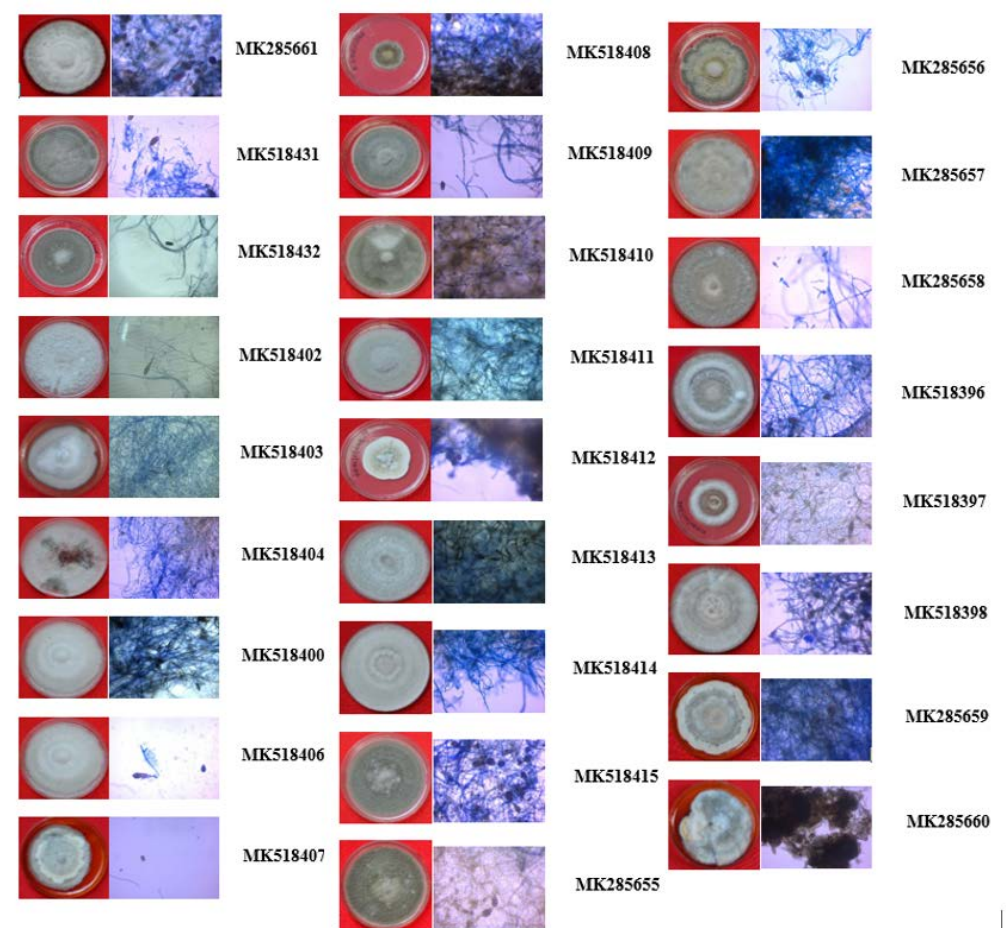

Fig. 3 - Cultivable entophytic fungi from Basella alba. The letters and numbers indicate the ITS accession numbers of the fungi. The mycelium and the spores were stained with lactophenol cotton blue. 


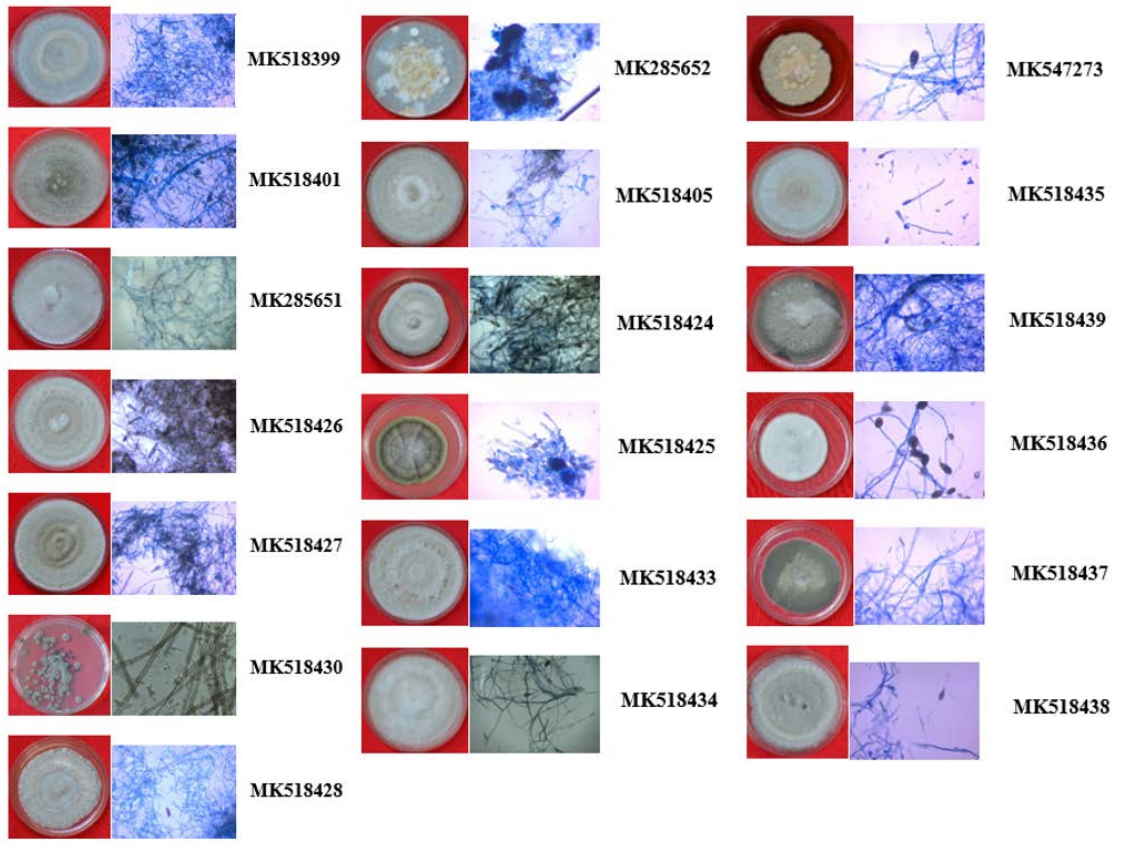

Fig. 4 - Cultivable entophytic fungi from Basella rubra. The letters and numbers indicate the ITS accession numbers of the fungi. The mycelium and the spores were stained with lactophenol cotton blue.

All the fungal sequences from both the plants were submitted to NCBI and the accession numbers and the organisms were mentioned (Tables 2, 3).

Table 2 Cultivable endophytic fungi on PDA from Basella alba.

\begin{tabular}{|c|c|c|c|c|c|c|}
\hline $\begin{array}{l}\text { ID given to } \\
\text { the } \\
\text { organism }\end{array}$ & $\begin{array}{l}\text { Genus and } \\
\text { Species }\end{array}$ & $\begin{array}{l}\text { Division/ } \\
\text { Phylum }\end{array}$ & $\begin{array}{l}\text { ITS } \\
\text { GenBank } \\
\text { accession } \\
\text { number }\end{array}$ & $\begin{array}{l}\text { Homologous } \\
\text { organism }\end{array}$ & $\begin{array}{l}\% \\
\text { homology }\end{array}$ & $\begin{array}{l}\text { Size } \\
\text { (bp) }\end{array}$ \\
\hline $\mathrm{BA} / \mathrm{L} / 1$ & $\begin{array}{l}\text { Alternaria } \\
\text { tenuissima }\end{array}$ & Ascomycota & MK285661 & $\begin{array}{l}\text { Alternaria } \\
\text { tenuissima strain } \\
\text { sp12 MH938073 }\end{array}$ & $99 \%$ & 552 \\
\hline $\mathrm{BA} / \mathrm{L} / 2$ & Alternaria sp. & Ascomycota & MK518431 & $\begin{array}{l}\text { Alternaria sp. } \\
\text { KY031981 }\end{array}$ & $99 \%$ & 535 \\
\hline $\mathrm{BA} / \mathrm{L} / 3$ & $\begin{array}{l}\text { Alternaria } \\
\text { alternata }\end{array}$ & Ascomycota & MK518432 & $\begin{array}{l}\text { Alternaria } \\
\text { alternate } \\
\text { MF422130 }\end{array}$ & $99 \%$ & 539 \\
\hline $\mathrm{BA} / \mathrm{L} / 4$ & $\begin{array}{l}\text { Alternaria } \\
\text { arborescens }\end{array}$ & Ascomycota & MK518402 & $\begin{array}{l}\text { Alternaria } \\
\text { tenuissima } \\
\text { MH237663 }\end{array}$ & $100 \%$ & 504 \\
\hline $\mathrm{BA} / \mathrm{L} / 5$ & $\begin{array}{l}\text { Alternaria } \\
\text { alternata }\end{array}$ & Ascomycota & MK518403 & $\begin{array}{l}\text { Alternaria sp. } \\
\text { KX011019 }\end{array}$ & $98 \%$ & 560 \\
\hline $\mathrm{BA} / \mathrm{L} / 6$ & Pleosporales sp. & Ascomycota & MK518404 & $\begin{array}{l}\text { Alternaria } \\
\text { tenuissima } \\
\text { MH237663 }\end{array}$ & $100 \%$ & 530 \\
\hline $\mathrm{BA} / \mathrm{L} / 7$ & Alternaria sp. & Ascomycota & MK518400 & $\begin{array}{l}\text { Alternaria sp. } \\
\text { KY031981 }\end{array}$ & $99 \%$ & 440 \\
\hline $\mathrm{BA} / \mathrm{L} / 8$ & $\begin{array}{l}\text { Alternaria } \\
\text { arborescens }\end{array}$ & Ascomycota & MK518406 & $\begin{array}{l}\text { Alternaria } \\
\text { tenuissima } \\
\text { MH237663 }\end{array}$ & $100 \%$ & 506 \\
\hline $\mathrm{BA} / \mathrm{L} / 9$ & Stemphylium sp & Ascomycota & MK518407 & $\begin{array}{l}\text { Stemphylium } \\
\text { solani } \\
\text { MH374556 }\end{array}$ & $99 \%$ & 549 \\
\hline
\end{tabular}


Table 2 Continued.

\begin{tabular}{|c|c|c|c|c|c|c|}
\hline $\begin{array}{l}\text { ID given to } \\
\text { the } \\
\text { organism }\end{array}$ & $\begin{array}{l}\text { Genus and } \\
\text { Species }\end{array}$ & $\begin{array}{l}\text { Division/ } \\
\text { Phylum }\end{array}$ & $\begin{array}{l}\text { ITS } \\
\text { GenBank } \\
\text { accession } \\
\text { number } \\
\end{array}$ & $\begin{array}{l}\text { Homologous } \\
\text { organism }\end{array}$ & $\begin{array}{l}\text { \% } \\
\text { homology }\end{array}$ & $\begin{array}{l}\text { Size } \\
\text { (bp) }\end{array}$ \\
\hline BA/L/10 & Pleosporales sp. & Ascomycota & MK518408 & $\begin{array}{l}\text { Pleosporales sp. } \\
\text { MG669157 }\end{array}$ & $100 \%$ & 526 \\
\hline BA/L/11 & $\begin{array}{l}\text { Alternaria } \\
\text { brassicae }\end{array}$ & Ascomycota & MK518409 & $\begin{array}{l}\text { Huperzia serrata } \\
\text { MK424421 }\end{array}$ & $99 \%$ & 506 \\
\hline BA/L/12 & $\begin{array}{l}\text { Alternaria } \\
\text { alternata }\end{array}$ & Ascomycota & MK518410 & $\begin{array}{l}\text { Alternaria } \\
\text { alternate } \\
\text { MH521178 }\end{array}$ & $99 \%$ & 546 \\
\hline BA/L/13 & $\begin{array}{l}\text { Alternaria } \\
\text { alternata }\end{array}$ & Ascomycota & MK518411 & $\begin{array}{l}\text { Alternaria } \\
\text { alternate } \\
\text { MF422133 }\end{array}$ & $99 \%$ & 554 \\
\hline BA/L/14 & $\begin{array}{l}\text { Microdiplodia } \\
\text { sp. }\end{array}$ & Ascomycota & MK518412 & $\begin{array}{l}\text { Microdiplodia } \\
\text { sp. MK247826 }\end{array}$ & $99 \%$ & 551 \\
\hline BA/L/15 & Alternaria sp. & Ascomycota & MK518413 & $\begin{array}{l}\text { Alternaria sp. } \\
\text { MF380509 }\end{array}$ & $99 \%$ & 540 \\
\hline BA/L/16 & $\begin{array}{l}\text { Alternaria } \\
\text { alternata }\end{array}$ & Ascomycota & MK518414 & $\begin{array}{l}\text { Alternaria } \\
\text { alternata } \\
\text { KX099664 }\end{array}$ & $99 \%$ & 542 \\
\hline $\mathrm{BA} / \mathrm{L} / 17$ & Pleosporales sp. & Ascomycota & MK518415 & $\begin{array}{l}\text { Pleosporales sp. } \\
\text { MG669157 }\end{array}$ & $100 \%$ & 526 \\
\hline $\mathrm{BA} / \mathrm{L} / 18$ & $\begin{array}{l}\text { Alternaria } \\
\text { tenuissima }\end{array}$ & Ascomycota & MK285655 & $\begin{array}{l}\text { Alternaria } \\
\text { tenuissima } \\
\text { HG798721 }\end{array}$ & $99 \%$ & 548 \\
\hline BA/L/19 & $\begin{array}{l}\text { Alternaria } \\
\text { tenuissima }\end{array}$ & Ascomycota & MK285656 & $\begin{array}{l}\text { Alternaria } \\
\text { tenuissima } \\
\text { HG798721 }\end{array}$ & $99 \%$ & 546 \\
\hline BA/L/20 & $\begin{array}{l}\text { Alternaria } \\
\text { alternata }\end{array}$ & Ascomycota & MK285657 & $\begin{array}{l}\text { Alternaria } \\
\text { alternata } \\
\text { MH938076 }\end{array}$ & $99 \%$ & 548 \\
\hline BA/L/21 & $\begin{array}{l}\text { Alternaria } \\
\text { alternata }\end{array}$ & Ascomycota & MK285658 & $\begin{array}{l}\text { Pleosporaceae } \\
\text { sp. } \\
\text { HM990181 }\end{array}$ & $99 \%$ & 549 \\
\hline BA/L/22 & $\begin{array}{l}\text { Alternaria } \\
\text { alternata }\end{array}$ & Ascomycota & MK518396 & $\begin{array}{l}\text { Alternaria } \\
\text { alternata } \\
\text { MH532479 }\end{array}$ & $100 \%$ & 480 \\
\hline BA/L/23 & $\begin{array}{l}\text { Alternaria } \\
\text { alternata }\end{array}$ & Ascomycota & MK518397 & $\begin{array}{l}\text { Alternaria sp } \\
\text { MK541638 }\end{array}$ & $100 \%$ & 480 \\
\hline BA/L/24 & $\begin{array}{l}\text { Alternaria } \\
\text { tenuissima }\end{array}$ & Ascomycota & MK518398 & $\begin{array}{l}\text { Alternaria } \\
\text { tenuissima } \\
\text { MK248473 }\end{array}$ & $100 \%$ & 491 \\
\hline BA/L/25 & $\begin{array}{l}\text { Alternaria } \\
\text { longipes }\end{array}$ & Ascomycota & MK285659 & $\begin{array}{l}\text { Alternaria sp } \\
\text { MH754629 }\end{array}$ & $99 \%$ & 553 \\
\hline BA/L/26 & $\begin{array}{l}\text { Stemphylium } \\
\text { lycopersici }\end{array}$ & Ascomycota & MK285660 & $\begin{array}{l}\text { Stemphylium } \\
\text { lycopersici } \\
\text { KX858849 }\end{array}$ & $99 \%$ & 560 \\
\hline
\end{tabular}

\section{Phylogenetic analysis}

While constructing the phylogenetic trees, the topology and branch length of the trees were estimated from a distance matrix. When one is using the average similarity (sequence) method, it is basic to obtain a distance matrix by comparing the whole genomes. A neighbour-joining tree without distance correction was obtained and represented in Figs 5, 6. 
Table 3 Cultivable endophytic fungi on PDA from Basella rubra.

\begin{tabular}{|c|c|c|c|c|c|c|}
\hline $\begin{array}{l}\text { ID given to } \\
\text { the } \\
\text { organism }\end{array}$ & $\begin{array}{l}\text { Genus and } \\
\text { Species }\end{array}$ & $\begin{array}{l}\text { Division/ } \\
\text { Phylum }\end{array}$ & $\begin{array}{l}\text { ITS } \\
\text { GenBank } \\
\text { accession } \\
\text { number }\end{array}$ & $\begin{array}{l}\text { Homologous } \\
\text { organism }\end{array}$ & $\begin{array}{l}\% \\
\text { homology }\end{array}$ & $\begin{array}{l}\text { Size } \\
\text { (bp) }\end{array}$ \\
\hline $\mathrm{BR} / \mathrm{L} / 1$ & $\begin{array}{l}\text { Alternaria } \\
\text { brassicicola }\end{array}$ & Ascomycota & MK518399 & $\begin{array}{l}\text { Alternaria } \\
\text { tenuissima } \\
\text { MK248473 }\end{array}$ & 100 & 486 \\
\hline $\mathrm{BR} / \mathrm{L} / 2$ & $\begin{array}{l}\text { Alternaria } \\
\text { alternata }\end{array}$ & Ascomycota & MK518401 & $\begin{array}{l}\text { Alternaria solani } \\
\text { MK551177 }\end{array}$ & $100 \%$ & 449 \\
\hline $\mathrm{BR} / \mathrm{L} / 3$ & Pleosporales sp. & Ascomycota & MK285651 & $\begin{array}{l}\text { Alternaria sp. } \\
\text { KY031981 }\end{array}$ & $99 \%$ & 552 \\
\hline $\mathrm{BR} / \mathrm{L} / 4$ & $\begin{array}{l}\text { Alternaria } \\
\text { tenuissima }\end{array}$ & Ascomycota & MK518426 & $\begin{array}{l}\text { Alternaria } \\
\text { tenuissima } \\
\text { MH938073 }\end{array}$ & $99 \%$ & 546 \\
\hline $\mathrm{BR} / \mathrm{L} / 5$ & $\begin{array}{l}\text { Alternaria } \\
\text { tenuissima }\end{array}$ & Ascomycota & MK518427 & $\begin{array}{l}\text { Alternaria } \\
\text { tenuissima } \\
\text { MH938073 }\end{array}$ & $99 \%$ & 529 \\
\hline $\mathrm{BR} / \mathrm{L} / 6$ & $\begin{array}{l}\text { Cladosporium } \\
\text { cladosporioides }\end{array}$ & Ascomycota & MK518430 & $\begin{array}{l}\text { Cladosporium } \\
\text { cladosporioides } \\
\text { KY400085 }\end{array}$ & $99 \%$ & 531 \\
\hline $\mathrm{BR} / \mathrm{L} / 7$ & $\begin{array}{l}\text { Alternaria } \\
\text { tenuissima }\end{array}$ & Ascomycota & MK518428 & $\begin{array}{l}\text { Alternaria } \\
\text { tenuissima } \\
\text { MH938073 }\end{array}$ & $99 \%$ & 508 \\
\hline $\mathrm{BR} / \mathrm{L} / 8$ & $\begin{array}{l}\text { Stemphylium } \\
\text { lycopersici }\end{array}$ & Ascomycota & MK285652 & $\begin{array}{l}\text { Stemphylium } \\
\text { lycopersici } \\
\text { KY290556 }\end{array}$ & $99 \%$ & 566 \\
\hline $\mathrm{BR} / \mathrm{L} / 9$ & Alternaria sp. & Ascomycota & MK518405 & $\begin{array}{l}\text { Alternaria sp. } \\
\text { KY031981 }\end{array}$ & $99 \%$ & 530 \\
\hline $\mathrm{BR} / \mathrm{L} / 10$ & Alternaria sp. & Ascomycota & MK518424 & $\begin{array}{l}\text { Alternaria } \\
\text { tenuissima } \\
\text { MH374277 }\end{array}$ & $98 \%$ & 571 \\
\hline BR/L/11 & $\begin{array}{l}\text { Alternaria } \\
\text { alternata }\end{array}$ & Ascomycota & MK518425 & $\begin{array}{l}\text { Alternaria sp. } \\
\text { KC139509 }\end{array}$ & $99 \%$ & 558 \\
\hline $\mathrm{BR} / \mathrm{L} / 12$ & Ascomycota sp. & Ascomycota & MK518433 & $\begin{array}{l}\text { Ascomycota sp. } \\
\text { KF160005 }\end{array}$ & $99 \%$ & 524 \\
\hline $\mathrm{BR} / \mathrm{L} / 13$ & Alternaria sp. & Ascomycota & MK518434 & $\begin{array}{l}\text { Alternaria sp. } \\
\text { KX011019 }\end{array}$ & 99 & 545 \\
\hline $\mathrm{BR} / \mathrm{L} / 14$ & Alternaria sp. & Ascomycota & MK547273 & $\begin{array}{l}\text { Alternaria sp. } \\
\text { MK541638 }\end{array}$ & 100 & 461 \\
\hline BR/L/15 & Alternaria sp. & Ascomycota & MK518435 & $\begin{array}{l}\text { Alternaria sp } \\
\text { KY031981 }\end{array}$ & 99 & 538 \\
\hline $\mathrm{BR} / \mathrm{L} / 16$ & Pleosporales sp. & Ascomycota & MK518439 & $\begin{array}{l}\text { Pleosporales sp. } \\
\text { MG669157 }\end{array}$ & $100 \%$ & 530 \\
\hline $\mathrm{BR} / \mathrm{L} / 17$ & Pleosporales sp. & Ascomycota & MK518436 & $\begin{array}{l}\text { Pleosporales sp. } \\
\text { MG669157 }\end{array}$ & $99 \%$ & 539 \\
\hline $\mathrm{BR} / \mathrm{L} / 18$ & $\begin{array}{l}\text { Alternaria } \\
\text { alternata }\end{array}$ & Ascomycota & MK518437 & $\begin{array}{l}\text { Alternaria } \\
\text { alternata } \\
\text { MG976287 }\end{array}$ & $100 \%$ & 519 \\
\hline $\mathrm{BR} / \mathrm{L} / 19$ & $\begin{array}{l}\text { Alternaria } \\
\text { alternata }\end{array}$ & Ascomycota & MK518438 & $\begin{array}{l}\text { Alternaria } \\
\text { alternata } \\
\text { KX099664 }\end{array}$ & $99 \%$ & 545 \\
\hline
\end{tabular}




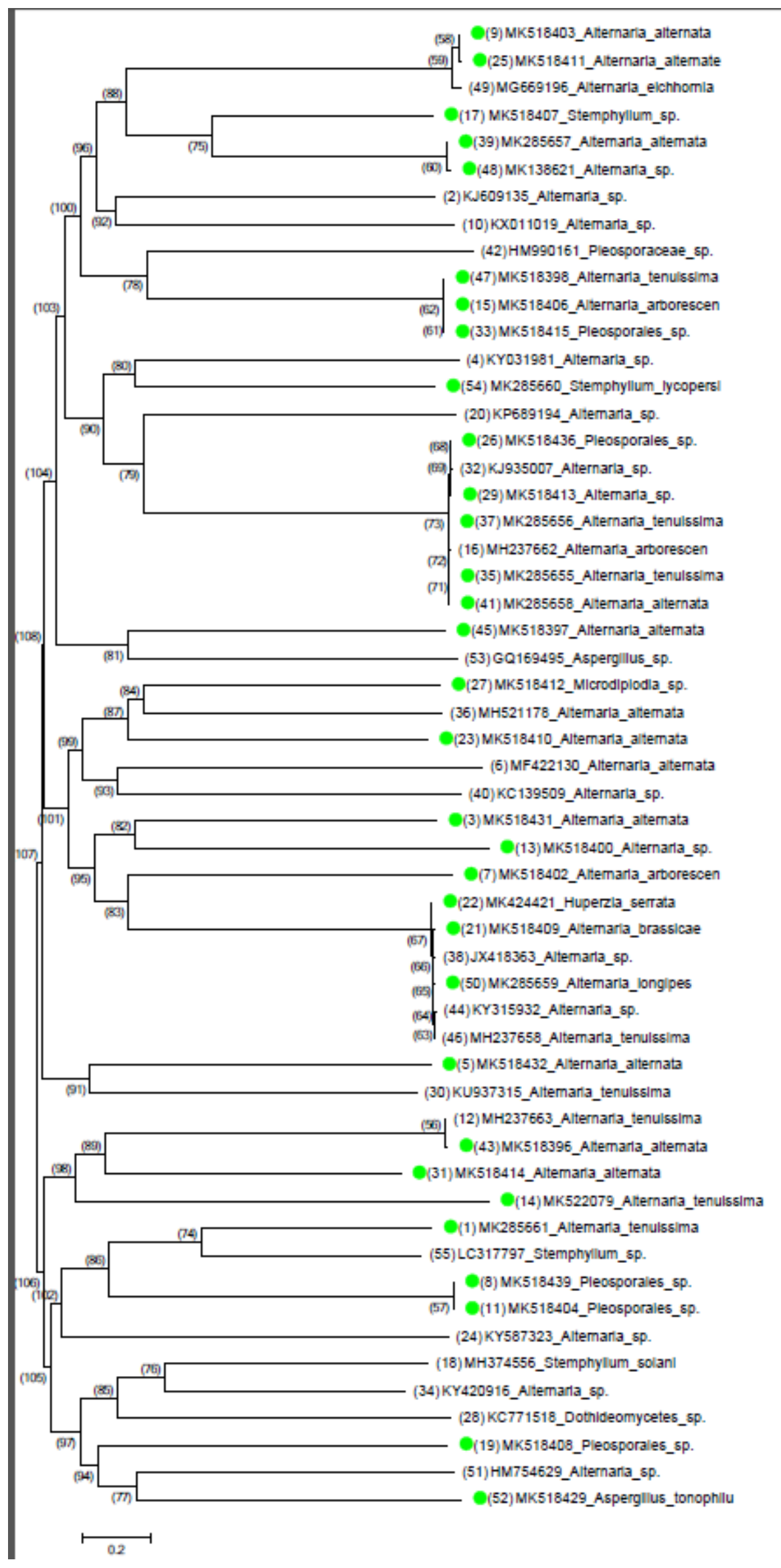

Fig. 5 - Phylogenetic tree of Basella alba concluded using Neighbor-Joining method for the organisms with ITS sequences. The endophytic fungi isolated in the present study from the selected plant are represented in green circles. 


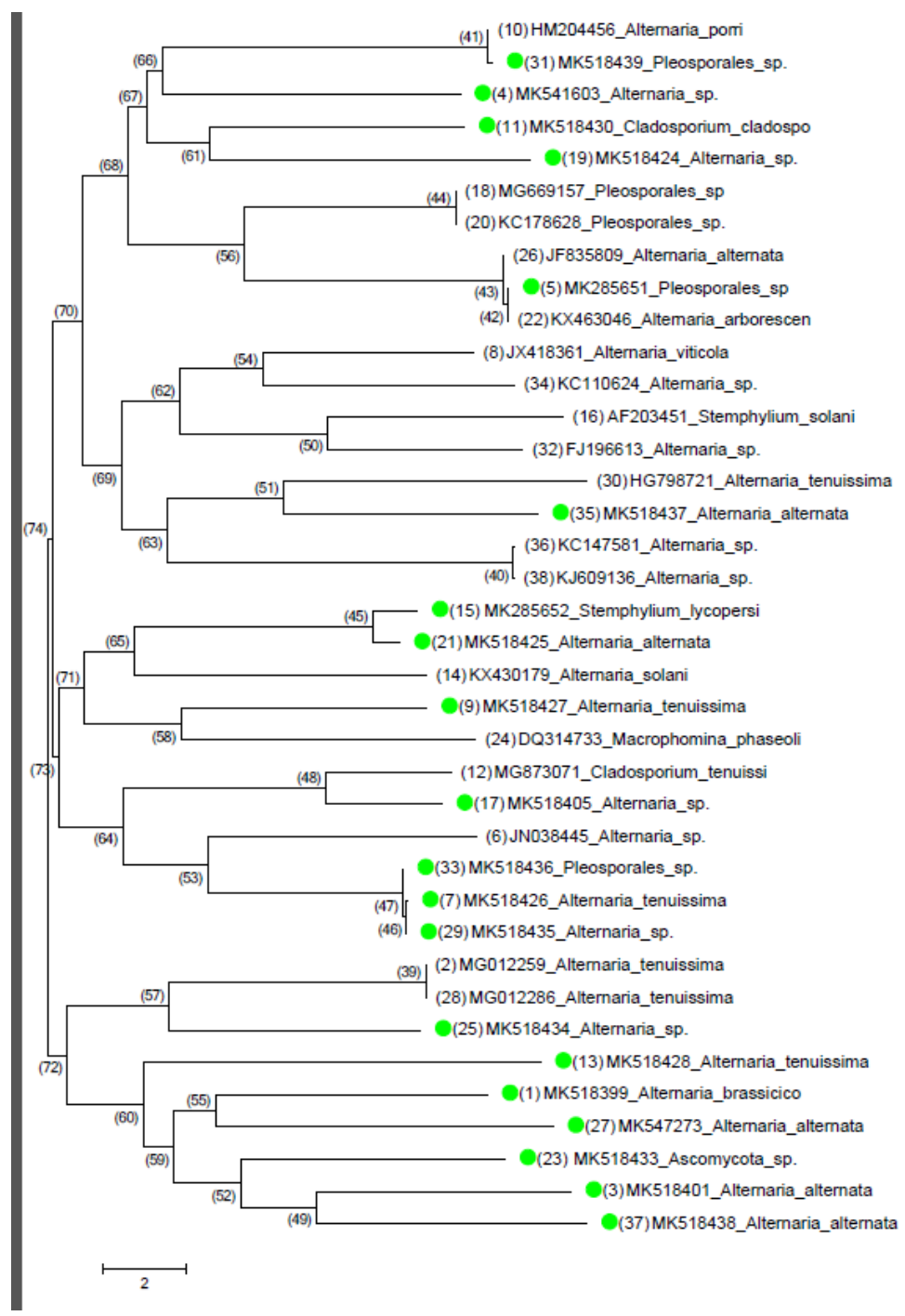

Fig. 6 - Phylogenetic tree of Basella rubra concluded using Neighbor-Joining method for the organisms with ITS sequences. The endophytic fungi isolated in the present study from the selected plant are represented in green circles.

\section{Seed borne fungal endophytes}

The fungal mycelium and spores were stained with lactophenol cotton blue. A total of six endophytic fungi have been identified by their spores (Figs 7, 8).

Our preliminary observation indicated that the vertically transmitted endophytic fungi are the dominant endophytes of the selected plants. There is a comprehensive distinction and harsh vertical endophytic fungal transmission from one generation to another.

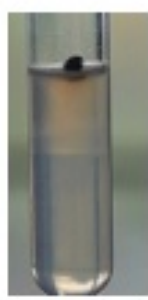

A

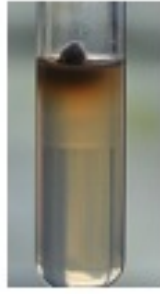

B

Fig. 7 - Scarified and surface sterilized seeds of Basella alba (A) and B. rubra (B) on 0.8\% autoclaved agarose 


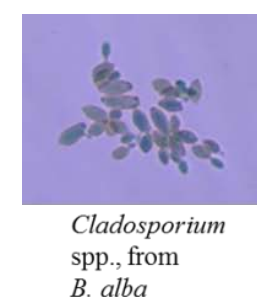

B. alba
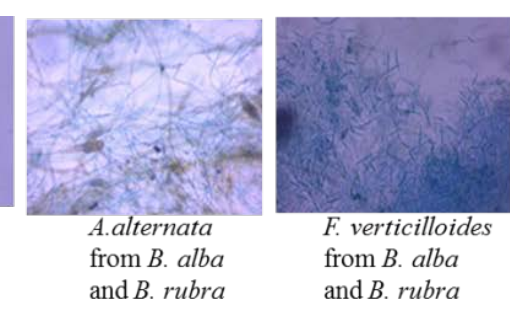
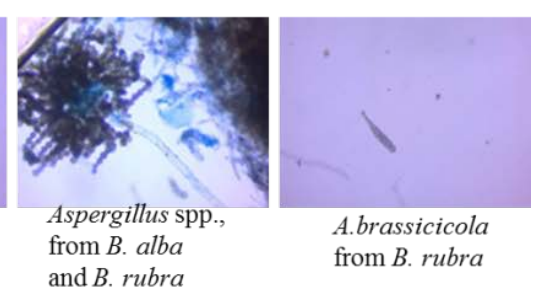

Fig. 8 - The morphology of seed born fungi from the selected plants stained with lactophenol cotton blue (Organisms were identified based on the spore morphology).

\section{Discussion and conclusions}

Endophytic fungi are non-infectious organisms in a healthy plant. In spite of their close relatedness to virulent pathogens, their virulence has limited pathogenic effects. Consequently, endophytes protect host plants from pathogenic microbes poisoned by mycotoxins (George 1988). The two mutualistic associations, constitutive and inducible, have independent effects on the respective host plant. In constitutive mutualism, the host and fungi have faithful association. Endophyte infects the ovule, propagates in the seeds in due course with the host's metabolic cost and develops all over the aerial parts of the plant, whereas, the inducible mutualist will not include in the seed and spread independently either through air, water or other sources. It is evident that inducible mutualist infect only the vegetative parts of the plant, inactive metabolically for an extended period of time, with a little fungal biomass. When the damage created by any herbivore or adverse conditions, they grow rapidly, produce toxins and provide new sites for infection (George 1988).

The traditional mycology says that most of the endophytic fungi, including both cultivable or non-cultivable, have been identified based on their morphological features like, spore, manner of the spore, color, odor, peridium morphology of the mycelium (Barseghyan \& Wasser 2010).

Based on morphology, Hema et al. (2015) recently reported a few endophytic fungi belong to taxa of Alternaria sp., Aspergillus sp., Chaetomium sp., Cercospora sp., Cladosporium sp., Corynespora sp., Curvularia sp., Nigrospora sp. and Monilia sp from B. rubra. In the present study, we isolated 47 (cultivable) endophytic fungi from Basella alba and B. rubra reported first time based on both morphology and ITS sequence. Morphologically though dissimilar, sequence analysis showed similarity.

In fungi, Dothideomycetes is considered to be the largest and ecologically much diversified class. They infect vast majority of plants, also human, tolerate to extreme environmental conditions (Ohm et al. 2012). Some class of fungi is reported as endophytes or epiphytes.

The markers for fungal studies should be shared by all fungi, have a high interspecific variation but not intra-specifically, the length should be appropriate to obtain proper amplification and sequences and more importantly, be possible to align across all the fungi. Even though no known markers meet all these mentioned requirements, components of the nuclear ribosomal repeat units are the most commonly used genetic markers for phylogenetic and taxonomic identification of fungi (Lindahl et al. 2013).

For finding out molecular ecology of fungi, the nuclear ribosomal ITS region is the only and most widely used for DNA sequence and fungal barcode. It helps to find out the genus to species and in some cases within species also (Schoch et al. 2012).

On an average, the length of the ITS region is approximately 500-600 bp, but varies from among the fungal populations. This ITS region has two variable spacers, and the intercalary 5.8S gene. The nuclear ribosomal repeat unit DNA genes are very much conserved across a large population of organisms. This makes them ideal criteria for general PCR primers. Though it is the scenario, the PCR amplified products also must contain enough variation to make it informative while making a phylogenetic analysis. This reason makes the mycologists to attract to ITS region, in particular. For example, Doilom et al. (2017) isolated fungal endophytes from Rhizophora 
apiculata in Thailand and established how may can be identified to species level based on ITS sequence data.

In addition to other phylloshpere endophytes, the vertical transmitted fungi play a critical role. It is evident that, Alternaria spp., Penicillium spp., Fusarium spp., helps to improve seed germination, seedling growth, Cladosproium spp., Aspergillus spp., help in antioxidant, antimicrobial potential (Shahzad et al. 2018)

In stringent vertical transmission, seeds of the two plants were infected with minimum of one common endophytic fungus. Every seed produced by B. alba and B. rubra may be distinctly infected with a distantly related fungi. The selected plants are tremendously occupied by the Dothideomycetes, Eurotiomycetes, a largest known class of filamentous fungi (Barret et al. 2015).

Based on the present investigation, we conclude that the genera Alternaria is predominant in both $B$. alba and B. rubra. It is a well-known that this genera is ubiquitous, occurs as pathogen (for example, Citrus sp., tomato, and potato (Peever et al. 2004), endophyte (Enhalus acoroides, Sapindus saponaria (Garcia et al. 2012, Sakayaroj et al. 2010).

In some instances, endophytism is a provisional condition, wherein a disease can or cannot evolve. It is a wise thinking of the stage of an endophyte rather than an endophyte as an organism. Most of the times, an endophyte boosts either abiotic, biotic stresses or any other useful event to the host plant. And the same endophyte causes disease when the plant becomes old or enters into senescence. It is understood that circumstances demand an endophyte as a pathogen or beneficial. Any asymptomatic fungi that harbour a host as an endophyte might be a weak pathogen, infectious strain or a resident waiting for suitable conditions to propagate. An isolate, like Alternaria spp, from a healthy plant, when sprayed on other plant may cause disease. The probable answer here is, may be the original host plant is immune to the disease caused by Alternaria or the other microbiota of the host modifies the fungal behaviour, that reduces the virulence. We need to pose a question to ourselves, what makes a microbe non-pathogenic phase rather than pathogenic phase? So there is a gap to be filled what causes this transition.

\section{Acknowledgements}

We thank Sri Devaraj Urs Academy of Higher Education and Research (SDUAHER) for funding. The work has been conducted at Department of Cell Biology and Molecular Genetics, SDUAHER and at Department of Plant Pathology, University of Agricultural Sciences, GKVK, Bangalore, supported by Indian National Science Academy Fellowship (INSA), New Delhi, India.

\section{Conflict of Interest}

Authors declare that there is no conflict of interest.

\section{References}

Barseghyan GS, Wasser SP. 2010 - Species diversity of hypogenous ascomycetes in Israel. Mycobiology 38(3), 159-165. Doi: 10.2307/1943154

Barret M, Briand M, Bonneau S, Préveaux A. 2015 - Emergence shapes the structure of the seed microbiota. Applied and Environmental Microbiology 81, 1257-1266.

Doi: 10.1128/aem.03722-14

Doilom M, Manawasinghe IS, Jeewon R, Jayawardena RS et al. 2017 - Can ITS sequence data identify fungal endophytes from cultures? A case study from Rhizophora apiculata. Mycosphere 8(10), 1869-1892. Doi:10.5943/mycosphere/8/10/11.

Faeth SH, Fagan WF. 2002 - Fungal endophytes: Common host plant symbionts but uncommon mutualists. Integrative and Compartive Biology 42(2), 360-368. Doi: 10.1093/icb/42.2.360.

Frank AC, Saldierna Guzman JP, Shay JE. 2017 - Transmission of bacterial endophytes. Microorganisms 5(4), 70. Doi: 10.3390/microorganisms5040070. 
Garcia A, Rhoden SA, Rubin Filho CJ, Nakamura CV. 2012 - Diversity of foliar endophytic fungi from the medicinal plant Sapindus saponaria L. and their localization by scanning electron microscopy. Biological Research 45, 139-148. Doi: 10.4067/S0716-97602012000200006.

George C. 1988 - Fungal endophytes in stems and leaves: From latent pathogen to mutualistic symbiont. Ecological Society of America, 69 (1), 2-9. Doi: 10.2307/1943154

Heinig U, Scholz S, Jennewein S. 2013 - Getting to the bottom of Taxol biosynthesis by fungi. Fungal Diversity 60 (1), 161-170. Doi: 10.1007/s13225-013-0228-7

Hema P, Murali M, Thriveni MC, Prathibha M. 2015 - Phytochemical analysis and antibacterial activity of endophytic fungi isolated from Basella rubra L. - A medicinal plant. Journal of Pure Applied. Microbiology 9 (4), 2971-2978.

https://www.researchgate.net/publication/289530914

http: //dx. doi.org/ 10.17504/protocols.io.jhmcj46. Isolation of endophytic fungi v1 (protocols.io.jhmcj46)

http://www.ncbi.nlm.nih.gov/

Jia M, Chen L, Xin HL. 2016 - A friendly relationship between endophytic fungi and medicinal plants: A systematic review. Frontiers of Microbiology 7, 906. doi: 10.3389/fmicb.2016.00906

Lindahl BD, Nilsson RH, Tedersoo L, Abarenkov K. 2013 - Fungal community analysis by highthroughput sequencing of amplified markers--a user's guide. New Phytologist 199 (1), 288299. Doi: 10.1111/nph.12243

Mishra Y, Singh A, Batra A, Sharma MM. 2014 - Understanding the biodiversity and biological applications of endophytic fungi: A review. J of Microbial and Biochemical Technology 1-11. Doi: 10.4172/1948-5948.S8-004

Ohm RA, Feau N, Henrissat B, Schoch CL. 2012 - Diverse life styles and strategies of plant pathogenesis encoded in the genomes of eighteen Dothideomycetes fungi. PLoS Pathogens 8 (12), e1003037. Doi: 10.1371/journal.ppat.1003037

Peever TL, Su G, Carpenter-Boggs L, Timmer LW. 2004 - Molecular systematics of citrusassociated Alternaria species. Mycologia. 96 (1), 119-134. Doi: 10.2307/3761993.

Rodríguez CE, Mitter B, Barret M, Sessitsch A. 2018 - Commentary: seed bacterial inhabitants and their routes of colonization. Plant and Soil 422(1-2), 129-134. Doi: 10.1007/s11104-0173368-9

Sakayaroj J, Preedanon S, Supaphon O, Jones EBG. 2010 - Phylogenetic diversity of endophyte assemblages associated with the tropical seagrass Enhalus acoroides in Thailand. Fungal Divers 42, 27-45. Doi: 10.1007/s13225-009-0013-9

Schoch CL, Seifert KA, Huhndorf S, Robert V. 2012 - Nuclear ribosomal internal transcribed spacer (ITS) region as a universal DNA barcode marker for fungi, PNAS 109 (16), 62416246. Doi: 10.1073/pnas.1117018109

Shade A, Jacques MA, Barret M. 2017 - Ecological patterns of seed microbiome diversity, transmission, and assembly. Current Opinion in Microbiology 37, 15-22.

Doi: 10.1016/j.mib.2017.03.010

Shahzad R, Khan AL, Bilal S, Asaf S. 2018 - What is there in seeds? Vertically transmitted endophytic resources for sustainable improvement in plant growth. Frontiers in Plant Science 9, 1-10. Doi: 10.3389/fpls.2018.00024

Sushanto G, Gitishree D, Sandeep KS, Han-Seung Shin, Jayanta KP. 2016 - Endophytes: A treasure house of bioactive compounds of medicinal importance. Frontiers in Microbiology 1, 1-8. Doi: 10.3389/fmicb.2016.01538

Tamura K, Peterson D, Peterson N, Stecher G et al. - MEGA5: molecular evolutionary genetics analysis using maximum likelihood, evolutionary distance, and maximum parsimony methods. Mol Biol Evol 2011; 28:2731-2739. doi: 10.1093/molbev/msr121. 